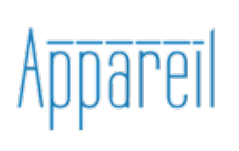

Appareil

14 | 2014

Esthétiques latino-américaines : penser à rebours

\title{
Poétique de l'enregistrement ou de l'enregistrement à la médiatisation
}

Javiera Medina

\section{(2) OpenEdition \\ 1 Journals}

\section{Electronic version}

URL: http://journals.openedition.org/appareil/2140

DOI: 10.4000/appareil.2140

ISSN: 2101-0714

Publisher

MSH Paris Nord

\section{Electronic reference}

Javiera Medina, «Poétique de l'enregistrement ou de l'enregistrement à la médiatisation », Appareil [Online], 14 | 2014, Online since 06 January 2015, connection on 30 July 2020. URL : http:// journals.openedition.org/appareil/2140; DOI : https://doi.org/10.4000/appareil.2140

This text was automatically generated on 30 July 2020

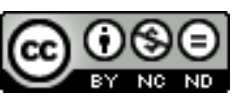

Appareil est mis à disposition selon les termes de la Licence Creative Commons Attribution - Pas d'Utilisation Commerciale - Pas de Modification 4.0 International. 


\title{
Poétique de l'enregistrement ou de l'enregistrement à la médiatisation
}

\author{
Javiera Medina
}

1 Le travail de Georges Didi-Huberman, et en particulier son livre Images malgré tout, se situe dans un contexte théorique spécifique. À la suite de l'exposition Photographies des camps de concentration et d'extermination nazis (1933-1999), la discussion sur la deuxième guerre mondiale en France a été relancée. Cette fois, le débat tournait spécifiquement autour de la monstration des photographies des camps. L'étendue de cette discussion qui, concernant d'abord l'Histoire, a atteint plus tard d'autres domaines de la pensée et de la création, est extrêmement vaste et a eu de multiples suites ces dernières années. Il s'avère donc impossible d'en rendre compte ici, ce qui n'est d'ailleurs pas dans notre intention.

2 Néanmoins, cette problématique est essentielle pour situer la question d'une esthétique de la résistance que nous élaborons au sein du milieu universitaire français, mais qui prend en considération les différences dans le traitement de la question dans les deux territoires géographiques - le Chili et la France - et dans ce que l'on pourrait appeler des territoires de la pensée (dont les frontières sont moins définies). La mise en contexte s'impose ainsi d'une théorie de la photographie élaborée en Amérique du Sud, et plus spécifiquement au Chili - où la recherche esthétique autour de la photographie s'est développée de manière particulièrement prolifique au cours de ces dernières années. Nous évoquons cet écart entre le chili et la France pour introduire les conditions dans lesquelles doit être posée la problématique des images de la dictature chilienne. Le colloque international Art et Politique, tenu les 2, 3 et 4 juin 2004 à l'Université ARCIS et à l'Université du Chili, à Santiago, abordait justement cette relation complexe, en divisant la période historique en question de la façon suivante : 1960-1973, 1973-1989, 1989-2004. Le deuxième segment correspondait intégralement à la période de la dictature. De nombreuses créations majeures de cette époque, radicales dans leur intention d'élaborer une critique du régime par la voie de l'art, ne sont visibles aujourd'hui que grâce à des enregistrements visuels et audiovisuels. Cependant, la photographie fut la grande absente de ce colloque sur la relation entre l'art et la 
politique au Chili dans la deuxième moitié du $\mathrm{xx}^{\mathrm{e}}$ siècle. Mais, au fond, pourquoi seraitil nécessaire de se poser la question de la photographie des actions, des performances, des happenings et des autres formes éphémères d'art réalisées à l'époque ? Cette pratique constituerait-elle un véritable chapitre pour l'histoire de l'art contemporain? Dans quelle mesure l'enregistrement photographique apporte-t-il des éléments pour penser une esthétique de la résistance contre une résistance aux images de la dictature?

En partant de l'esthétique de l'enregistrement proposée par François Soulages ${ }^{1}$, nous essayerons de penser une poétique de l'enregistrement. Sur la base de quelques photos de performances et d'actions artistiques, mais aussi de manifestations politiques des associations des familles des disparus et des individus engagés contre la dictature, nous essayerons de réfléchir à la prégnance visuelle de ces images aujourd'hui et à leur portée politique. Nous nous concentrerons sur deux axes principaux : d'une part, la question de la trace et de l'archive et, d'autre part, celle de l'abstraction. Ces deux questions sont présentes dans le travail de ces photos. Si l'argentique et le précaire font partie de leur esthétique, elle semble dépendre aussi de leur réception aujourd'hui, ainsi que des formes dans lesquelles elles ont été publiées ou diffusées, archivées ou pas. Le passage de la photographie comme moyen, à la photographie comme fin proposé par François Soulages serait aussi le fondement d'un travail esthétique de la photographie sur l'agir contestataire. La trace d'un acte révolté, artistique, deviendrait ainsi une condition de l'existence de l'œuvre par sa persistance. Or, dans le cas spécifique des photos de la dictature, il serait question aussi d'un passage de la manifestation politique à la manifestation artistique, et de l'effacement des frontières entre les deux par la voie d'un désir de visibilité des « corps manifestants ».

\section{Le photographe comme « enregistreur » : l'œil performant}

4 L'écrivain Diamela Eltit réalise en 1980 la performance Zone de douleur dans le quartier pauvre Maipu, à Santiago. Elle se présente avec les bras brûlés et pleins de coupures. Une lecture à haute voix fait également partie de la mise en scène : il s'agit de certains passages de son roman Lumpérica ${ }^{2}$, qui sera publié trois ans après. Le lieu choisi pour cette action est un bordel. Après la lecture, l'artiste lave le trottoir devant la maison close. Entretemps, une photo de son visage est projetée sur l'un des murs du bordel. L'enregistrement audiovisuel et photographique de cette action a été réalisé par Lotty Rosenfeld. Diamela Eltit a également participé au montage de la vidéo. Or, celle-ci est rarement montrée, le public ayant davantage accès à des images fixes - des photogrammes de la vidéo en couleurs ou des photographies en noir et blanc de la performance. Mais ces images ne représentent qu'un dispositif parmi ceux utilisés dans cette œuvre, à savoir la forme littéraire, orale et écrite, et la performance. L'image fixe serait à la fois une partie de l'action - le portrait photographique projeté de l'artiste -, un enregistrement de la performance - une trace de celle-ci - et une archive. Et, finalement, elle constituerait une sorte d'illustration de la performance : c'est le cas des deux photos publiées dans l'édition de Lumpérica, l'une sur la page de couverture et l'autre sur la p. 141 de la première édition.

5 Pourquoi partirions-nous de cette œuvre pour penser une poétique de l'enregistrement? Tout d'abord, en raison de l'espace choisi pour mettre en scène cette 
performance - un quartier marginal, un bordel -, et de la violence que cette action expose. Cet élément n'est pourtant pas exclusif à l'œuvre de Diamela Eltit: de nombreux exemples existent de performances et happenings d'artistes qui développent ce que l'on pourrait désigner comme une « mimique » de la violence ${ }^{3}$. Un autre élément viendrait donner un « cadre " à la pièce : il s'agit de la période pendant laquelle elle a lieu - la dictature. Cette mimique acquiert une autre dimension lorsque le spectateur prend conscience qu'il y a un revers de cette image: les individus qui seraient effectivement torturés sous le régime dictatorial et dont l'image resterait - reste invisible. L'importance de l'enregistrement photographique viendrait alors du fait qu'il constitue la trace - c'est ainsi qu'il est conçu - d'un acte qui a eu un caractère «sacrificiel », irrépétible, unique. Cet aspect presque rituel de l'œuvre, qui symbolise le sacrifice humain en vue de la rédemption de toute une société vivant dans la peur dans ce cas spécifique, il s'agit d'une partie de la société, celle des dissidents du régime - donne à son tour une allure particulière aux photographies. En effet, il les "charge " d'une résonance mystique. Une dramatisation se produit à travers ces images en noir et blanc et en couleurs, contrastées et peu nettes. Elles sont d'une certaine manière commentées, signalées, « légendées » par le contexte complexe qui les entoure - acte littéraire, œuvre littéraire, régime dictatorial.

6 Mais enfin, quelle serait la place et la position du photographe dans toute cette construction? Le photographe comme enregistreur y participe-t-il ? Si oui, contribue-til à l'élaboration d'une esthétique de l'enregistrement? Est-il un observateur voyeuriste - de la scène de douleur de l'artiste, ou fait-il partie de cette zone de douleur désignée par l'œuvre? Le photographe est ici présent en tant qu'enregistreur, c'est-à-dire que, par définition, sa place est derrière l'appareil. Néanmoins, il participe à l'espace de l'œuvre qu'il segmente et rythme à travers le déclic photographique ou celui de la prise de vue audiovisuelle. Une sorte de dynamique s'installe alors entre le corps enregistreur qui regarde et « suit ", et le corps performeur qui est conscient de ce regard. La question de la trace se définit dans ce rapport médiatisé par l'appareil. À ce propos, on pourrait s'interroger sur la différence qui existe entre les diverses images de l'œuvre, entre les divers angles de vue ou perspectives photographiques. La position variable du photographe - sa distance ou sa proximité, par exemple - fait que les images se complémentent les unes les autres. Elles transmettent chacune un sens spécifique, qui est à la fois un écho de la configuration globale de l'œuvre et une création indépendante.

7 Ces photos constituent aujourd'hui une archive. L'archive de l'œuvre? L'œuvre? Comment cette dernière peut-elle prendre forme dans l'actualité si ce n'est à travers la reprise et le «montage» de ces images? Pourquoi ces photos rendraient-elles compte d'une poétique de l'enregistrement? Avec Georges Didi-Huberman, nous insisterons sur la construction qu'exigent ces photographies, afin de nous " poser la question de savoir ce que ces images trahissent plutôt que de nous interroger d'abord sur ce qu'elles montrent $»^{4}$. Pour ce faire, il faudrait interroger une poétique de l'image enregistrée, en l'occurrence de la photo. Dans les premières pages du livre cité, François Soulages se réfère à la photographie dans ces termes : « [...] "Un poète, écrit René Char, doit laisser des traces de son passage, non des preuves. Seules les traces font rêver". Qui peut encore croire que la photo est une preuve? Une photo est une trace, c'est pourquoi elle est poétique $»^{5}$. 
8 Ces photographies seraient ainsi des traces de la performance de Diamela Eltit. Si l'on prend en compte le roman qui se rapporte à ce travail, on observe qu'il se trouve à la fois avant la réalisation, en tant qu'inspiration de la performance (il apporte le cadre et l'esthétique de l'action), dans l'action, en tant que déclamation orale d'un work in progress (le roman à ce moment-là inachevé et la performance), et après cette manifestation, en tant que trace constituée par un livre écrit et publié qui intègre l'expérience de l'«acte poétique». La photographie, quant à elle, ne serait ni une illustration du roman - ou de l'esthétique proposée par le roman- ni une représentation de la performance. Elle viendrait plutôt inscrire l'action dans une visibilité spécifique, en montrant le corps de l'artiste qui s'exprime et qui se manifeste dans les rues de la ville assiégée. En cela, elle libère ce corps par la voie de sa visualisation. Le dispositif photographique opère alors un prolongement de l'œuvre et atteste inconsciemment aussi d'un « état » de l'art en dictature.

9 L'œil de Lotty Rosenfeld devient performant, c'est-à-dire actif et indépendant, en ce qu'il propose un regard distancé, donnant à la fois une vision du geste de l'artiste et de l'espace qui l'entoure ${ }^{6}$. La photo met en scène la zone de douleur, elle la délimite plus clairement que le déplacement de l'artiste elle-même. Car la zone de douleur n'est pas la blessure, mais le regard posé sur la blessure : son exhibition. Cette extériorisation de la douleur, cette mimique que nous avons citée plus haut, est tracée par le regard de la photographe. Le geste qui y est fixé - le corps penché pour accomplir le travail de nettoyage -, exprimant une certaine humilité et une concentration, donne à l'ensemble une allure presque mystique de rituel hétérodoxe. L'émotion qui se dégage de ces photos provient de l'enjeu qu'entend réaliser une telle manifestation à ce moment-là au Chili. Le contexte est celui d'une société habitée par la peur, mais qui commence à développer dans cette période - au début des années 1980 - un travail soutenu de contestation fondé précisément sur la manifestation publique. Finalement, la mise-enscène proposée par Diamela Eltit nous est transmise par le regard de Lotty Rosenfeld, à travers des images fixes et des images en mouvement.

10 La problématique de la réception de ce travail tient à sa complexité, et aussi à l'abstraction qu'il mobilise. Comme si l'invisible l'emportait ici par-dessus le visible, et comme si la métaphore devenait le dernier refuge d'un art politique. L'enregistreur ne se concentrerait alors pas exclusivement sur les corps et les paysages, dans un souci du détail formel, mais plutôt sur le geste qui condense une image poétique. Il cherche non pas la simple médiatisation de la scène, mais son inscription contestataire, dans un contexte de censure invisibilisante.

11 La survivance de l'œuvre dépendrait, dans une certaine mesure, de la conservation des photographies. Une poétique de l'enregistrement serait liée précisément à cette précarité de l'action devenue œuvre, parmi d'autres facteurs, par le geste photographique, par l'œil performant du photographe. Une esthétique de la résistance devrait ainsi intégrer une réflexion de ce type afin de pouvoir penser, aux antipodes d'une résistance aux images, une prégnance non-critique de ces images de la douleur, qui risquerait d'enlever à ces photos leur charge politique originale. Dans ce cas, la diffusion de ces images trahirait leur origine même, qui est essentiellement politique non seulement en tant que contestation de la dictature, mais aussi comme une forme d'art cherchant à se démarquer des formes prescrites de représentation.

12 Dans son livre Mal d'archive, Jacques Derrida postule que « le principe archontique de l'archive est aussi un principe de consignation, c'est-à-dire de rassemblement $»^{7}$. Un 
peu plus loin, il écrit «Nul pouvoir politique sans contrôle de l'archive, sinon de la mémoire. La démocratisation effective se mesure toujours à ce critère essentiel : la participation et l'accès à l'archive, à sa constitution et à son interprétation $»^{8}$. Ces dernières années, le Chili a vécu une activité intense relative à l'archive de la dictature. La construction ou le développement d'espaces consacrés à cette période tels que le Musée de la mémoire et des droits de l'Homme, le Centre culturel Villa Grimaldi, le Museo de la Solidaridad Salvador Allende et le Centro Cultural La Moneda, parmi d'autres, a généré une intensification du travail de récupération, de sélection et de diffusion des images de la dictature. La publication de livres de photographie ou d'autres sortes de documents faisant partie de ces archives - tels qu'affiches, pamphlets, dessins, etc. -, ainsi que les émissions de télévision comme Chili, les images interdites, et les sites sur Internet, constituent les médias de diffusion les plus répandus de l'archive de la dictature.

Un décalage est pourtant perceptible entre, par exemple, l'infrastructure d'une construction telle que le Musée de la mémoire et des droits de l'Homme, et son archive photographique. Dans certains cas, l'archive se fonde sur des dons des particuliers, qui apportent leurs documents personnels. Mais, dans le cas de la photographie, la question est particulièrement problématique : elle serait, à nos yeux, la conséquence d'une absence de discussion autour d'une théorie de la photographie, qui exigerait justement un accès à l'archive et un travail d'interprétation de celle-ci. Ce processus d'élaboration de l'archive semble extrêmement important car il définit en quelque sorte une politique de l'archive, qui peut prendre ou ne pas prendre en compte des éléments tels que les photographies des performances réalisées en dictature ou les photographies des manifestations contre la dictature. Une pensée de l'enregistrement serait à la base d'une telle démarche, qui permettrait de mettre en question la (les) sélection(s), la pertinence de chaque archive, la séparation des archives en différents espaces ou leur rassemblement, et, éventuellement, la visibilité et le partage des dites images.

Si l'archive implique le rassemblement, la sélection et l'organisation des images, ainsi que leur diffusion, elle implique aussi un regard et une vision, voire une politique des images, de l'archive et de l'accessibilité à cette dernière. En résumé, la mise en archive des photos de la dictature qui manifestent un œil performant exige un débat et un travail théorique sur leurs conditions de réalisation et de conservation, ainsi que sur le « destin de (ces) images ». En paraphrasant Jacques Rancière, nous dirons :

Ainsi s'est tissée une solidarité entre les opérations de l'art, les formes de l'imagerie et la discursivité des symptômes. Cette solidarité s'est encore compliquée, à mesure que les vignettes de la pédagogie, les icônes de la marchandise et les étalages marchands désaffectés ont perdu leurs valeurs d'usage et d'échange. Car elles ont alors reçu en contrepartie une valeur nouvelle d'image qui n'est rien d'autre que la double puissance des images esthétiques : l'inscription des signes d'une histoire et la puissance d'affection de la présence brute qui ne s'échange plus contre rien. ${ }^{9}$

Si l'acte de photographier, en deçà de la publication des images, est déjà, en lui-même, un acte politique, le résultat, la photographie, est un fragment de contestation arraché à la censure. Finalement, l'enregistrement est, d'une part, trace d'un certain mouvement et, d'autre part, signe d'un déplacement du regard. Nous passons ainsi d'un enregistrement $\mathrm{du}$ corps manifestant la violence au moyen de l'art, à un enregistrement des « corps politiques » actifs des manifestants dans la rue. 


\section{L'enregistrement et l'acte politique. Photographie des manifestations} permis le retour de la démocratie. Le travail des photographes s'insérait dans une configuration incluant, d'une part, manifestations et actes organisés par les associations d'opposition (Familles des disparus, Contre la torture Sebastian Acevedo, Femmes pour la vie, etc.) et, d'autre part, le travail naissant des médias de gauche. Ces trois éléments qui se retro-alimentaient - manifestations, médias et photographie - ont permis de donner une visibilité au mouvement d'opposition, aussi bien à l'étranger qu'à l'intérieur du pays, en détournant la censure. Les manifestations appelées protestas étaient parfois le résultat de longs processus de production où les différentes associations se mettaient d'accord pour convenir d'un lieu et d'une date pour le rassemblement. remettaient au travail de reconstitution progressive de l'exercice politique en élaborant un projet de retour à la démocratie, la forme des manifestations évoluait en rendant compte d'une libération de la capacité expressive des citoyens. La visibilité de ces actions courageuses était fondamentale, au vu du silence de la presse officielle et de la télévision. Les photographes de presse d'opposition étaient présents lors des manifestations, tout comme les caméramans - qui étaient conscients que leurs enregistrements ne passeraient pas à la télévision mais seraient diffusés par la presse étrangère et par quelques rares médias audiovisuels indépendants tels que Teleanalisis (Téléanalyse) ${ }^{10}$. Ils constituaient ainsi une archive qu'il fallait conserver pour pouvoir la montrer une fois que la dictature serait finie. Leur présence était à tel point importante, qu'Ana Gonzalez, membre de l'association des Familles des détenus disparus, affirme dans le témoignage recueilli par le film La Cité des Photographes : « les actions se faisaient pour que les photographes viennent. Il s'agissait de beaucoup plus que ça, mais les photographes étaient indispensables, et on se mettait d'accord avec eux pour qu'ils soient là, et ils étaient là, et l'information ne filtrait pas, car la police arrivait toujours après $»^{11}$.

18 Ces paroles rendent compte d'une complicité entre manifestants et photographes qui, d'une certaine manière, les place tous deux en acteurs de ces manifestations. En effet, pour que ces dernières deviennent visibles, elles devaient être montrées, la photo accomplissant le travail de diffusion de ces actes. La plupart du temps, ces enregistrements se faisaient donc dans l'urgence, avec la conscience de l'arrivée imminente de la police. La prise de vue, rapide, ne constitue pas une image élaborée ; on dirait qu'il s'agit d'une photo « sans-art $»^{12}$

Il serait donc question ici d'une approche esthétique des images sans qualité. Le regard du photographe serait alors compris comme point commun entre l'art et le document, car c'est lui qui enregistre aussi bien les manifestations de l'art que les manifestations politiques. En dépit de leurs différences, ces manifestations deviennent similaires à certains égards dans le contexte de la dictature. Le travail de l'art en dictature et sur elle semble tendre à éviter la disparition de quelque chose qui ferait l'objet d'une dénégation de la dictature - ses crimes, mais aussi la résistance qui s'y opposait. La photographie occuperait une place centrale dans la restitution ou dans la création d'une image actuelle de la dictature, car elle serait toujours la représentation d'un geste 
de résistance à l'oubli, plutôt qu'une représentation de la dictature en tant que telle. Les multiples photographies des manifestations publiques d'opposition dévoilent, d'une part, une évolution rendant ces dernières de plus en plus séduisantes et massives et, d'autre part, elles soulignent un regard qui, au-delà des conditions de la prise de vue, devient progressivement plus personnel.

Cette manière de voir l'enregistrement contraste fortement avec celle qui sous-tend les images de la presse locale dans l'actualité, où les mêmes images des mêmes événements se répètent avec de moindres variations, dans une espèce d'homogénéisation du regard. Il y aurait donc un élément esthétique à retracer du côté des formes développées par les corps manifestants pour accéder à une visibilité majeure, et aussi dans la présentation et la lecture des photographies de ces actions aujourd'hui. La présence de ces photographies aujourd'hui dans des films tels que la Cité des photographes, dans des livres et des monographies - notamment le travail de Gonzalo Leiva sur l'association des Photographes indépendants $\mathrm{AFI}^{13}$-, dans des expositions et aussi dans la presse, sur Internet, etc., demande un travail de mise en lumière de ces pratiques et de mise en question de la diffusion des photographies elles-mêmes.

21 Les clichés qui existent des manifestations contre la dictature sont nombreux. Certaines de ces images peuvent être qualifiées de fortes. Elles visent, au-delà des conséquences immédiates ou à long terme que ces manifestations ont pu avoir, cet espace d'abréaction que génère la possibilité d'une manifestation communautaire. À ce point de notre recherche, nous revenons à la question de la résistance aux images et des images de la résistance, pour préciser la distinction entre la première et les secondes, et surtout pour visualiser le passage de celle-ci à celles-là du point de vue de la réception. Ces images montrent précisément une résistance ; c'est en cela qu'elles ne provoquent pas de résistance, car elles ne sont pas traumatiques.

Pour mieux cerner la question, il est nécessaire d'inclure dans ce corpus d'images des manifestations qui sortent de la logique de la protesta massive, pour se concentrer sur une visibilité intensifiée par une mise en scène des corps dans l'espace public, c'est-àdire par des performances des corps politiques manifestants. Une de ces actions correspond à ce qu'on a appelé La Cueca sola. Traditionnellement, la cueca, danse typique chilienne, est une danse à deux - une femme et un homme. La mise en scène des femmes des disparus se réalise de la manière suivante : une veuve, fille ou mère de disparu, danse la cueca toute seule, la plupart du temps habillée en noir ou avec une chemise blanche et une jupe noire. Elle porte la photo du disparu accrochée sur la poitrine, soulignant ainsi l'absence de son compagnon disparu et son deuil inachevé. La première manifestation de ce type a eu lieu lors d'un acte de célébration de la journée de la femme le 8 mars 1978 au théâtre Caupolican, à Santiago.

Cette Cueca sola a été reprise par la suite par le couple d'artistes Les yeguas del Apocalipsis (Les juments de l'Apocalypse), dans une performance qui présentait une version extrême de cette action. Les artistes ont dansé ensemble - deux hommes revendiquant leur homosexualité - sur une carte d'Amérique recouverte de verres de bouteilles de Coca Cola cassées. La performance a eu lieu le 12 octobre 1989 à midi au siège de la Commission chilienne des droits de l'Homme. Nous voyons dans ces deux photographies le passage d'un acte politique à une action artistique: le premier consiste en la dénonciation des disparus, tandis que la seconde insiste sur cette dénonciation tout en ajoutant d'autres revendications - comme l'opposition à l'homophobie -, et en intégrant des éléments qui symbolisent le problème économique 
d'un pays qui a introduit violemment un système économique néolibéral. Une troisième image vient s'ajouter aux deux photos : il s'agit de la reconstitution de cette action pour le spot télévisé qui fait partie de la campagne référendaire pour le «non $»^{14}$ à la dictature. Cette dernière aura comme résultat la fin de la dictature et le retour à la démocratie à travers les élections présidentielles en décembre 1989.

Dans la version télévisée de cette action, la mise en scène devient plus illustrative, c'est-à-dire que le montage des images introduit d'abord les femmes qui se présentent en disant leurs noms et leur lien de parenté avec la personne disparue : mère, femme, fille, etc. Ensuite, s'ajoutant à l'image de la femme qui danse seule, une voix off dit: "Que cela n'arrive plus jamais, ça dépend de vous", "plus jamais des disparus". L'action politique a donc été décodée par les mécanismes de l'image publicitaire. À la différence de l'enregistrement de la performance Zone de douleur et de celui des manifestations contre la dictature, la photo de la Cueca sola est à la fois une image d'archive et une image qui se réactualise à chaque fois qu'elle a lieu. C'est-à-dire que cette image se répand et dépasse d'une certaine manière les limites de l'enregistrement. Car ce qui prévaut, c'est le geste d'une femme, non d'une femme en particulier, mais de n'importe quelle femme ayant un lien de parenté avec un disparu et qui, à travers la mise en scène de son corps en mouvement, évoque la disparition de son compagnon de danse. Il faudrait signaler aussi que l'identité de la créatrice de cette manifestation est toujours restée au deuxième plan, à la différence, par exemple, de la « signature » de Diamela Eltit dans la performance étudiée plus haut.

Les trois images des trois versions de la Cueca sola nous invitent à poser la question de leur lecture aujourd'hui, dans le contexte de l'archive. La diversité de ces images photo de performance faite par une artiste, photo de manifestation prise par un photoreporter engagé, photo d'action politique faite par un photoreporter - nous confronte à la question de l'auteur de l'image, ainsi qu'à celle des conditions de sa prise de vue et de ses possibilités de lecture et de diffusion. Ce groupement d'images, qui rend compte du principe archontique qu'évoque Jacques Derrida, est en lui-même complexe du seul fait du regroupement de ces images pour observer leurs relations et leurs différences possibles, avec les réflexions qui en découlent. La visibilité de ces images étant fondamentale, le pas suivant consiste précisément en une démocratisation et en une ouverture effective de l'archive à la parole et au débat. Par leur complexité et par la dialectique qu'elles engendrent ainsi posées, les unes à côté des autres, ces images nous permettent de postuler une poétique de l'enregistrement fondée notamment sur leur condition de trace. Elles obligent ainsi le récepteur à se positionner dans le contexte de la prise de vue, et par rapport à une certaine difficulté de lisibilité aujourd'hui résultant des problèmes liés au travail défaillant de mémoire qui caractérise le Chili des années postérieures à la fin de la dictature.

\section{Poétique de l'enregistrement ou de l'enregistrement à la médiatisation}

Nous avons déjà évoqué l'approche de l'archive proposée par Jacques Derrida dans son livre Mal d'archive. Ces réflexions nous intéressent car elles partent d'une lecture psychanalytique de l'archive. La question de la mémoire de la dictature chilienne en ce qui concerne la résistance aux images du trauma - considéré comme un nœud complexe et irrésolu - pourrait être pensée à l'aide des deux éléments suivants. Le 
premier est la question, plus générale, de l'archive : qu'est-ce qu'une archive? Mais aussi, que suppose la fabrication d'une archive en termes de destruction, de discours sur la mémoire, d'évaluation et d'analyse des pièces à conserver (ou pas) ? Le second concerne la diffusion de cette archive, la question de la manière dont les images se montrent ou ne se montrent pas - sont-elles accessibles ou séduisantes pour une population qui ignore les événements de la période, notamment les nouvelles générations?

La référence aux racines grecques du concept d'analyse dans le livre Les résistances de la psychanalyse, nous a permis de penser avec Derrida la question de l'évaluation et du regard posé sur ces pratiques pendant et après la dictature. Les observations et les interprétations que nous pouvons faire de ce matériel d'archive étant infinies, elles semblent aujourd'hui réduites à une expression minimale vis-à-vis de la densité qu'elles portent et de la complexité de leur langage. Nous pensons ici le langage en termes de nouvelle forme d'expression " contre » la censure, c'est-à-dire une expression à la fois critique et suffisamment dissimulée pour contourner cette dernière. Pendant les dixsept ans de dictature, certaines personnes ont effectivement résisté, elles se sont battues et ont survécu à l'oppression; quelques images en rendent compte, en nous apportant des éléments ou des aperçus de ce que pouvait être le quotidien de la répression.

La question qui surgit aujourd'hui, à une époque où le coup d'État et la dictature semblent si loin et en même temps si présents, est la suivante : comment serait-il possible de surmonter la résistance qui bloque le travail de mémoire ? Comment est-il envisageable pour le spectateur de renouer le lien avec ce passé pour pouvoir s'emparer de ces images, se les approprier ? Ou est-il nécessaire de trouver la «bonne distance»? En reprenant le postulat de Georges Didi-Huberman dans son livre Quand les images prennent position: "Ainsi, distancier serait montrer en montrant que l'on montre et en dissociant par là - pour en mieux démontrer la nature complexe et dialectique - ce que l'on montre. En ce sens, donc, distancer, c'est monter, c'est-à-dire disjoindre les évidences pour mieux ajointer, visuellement et temporellement, les différences $»^{15}$.

Mais comment montrer le traumatisme, comment reconstruire à partir de fragments ? Le montage requiert une "substance » qui servirait à remplir les «écarts » entre les images : les « fissures d'image ». Pour montrer la violence dans toutes ses expressions possibles - une violence qui, dans le cas de la dictature chilienne, était censée s'exercer "légalement»-, et pour restituer quelque chose d'une histoire pleine de conflits visibles et invisibles, il est nécessaire d'insister sur les images-vestiges de la période, sur les images nouvelles qui s'y confrontent, et sur les traces que dévoilent les usages de l'image, c'est-à-dire ses censures, ses imprécisions, ses occultations, ses montages. Ce faisant, une résistance est fondamentale pour mettre en place un travail de mémoire qui, ne changeant pas forcément notre rapport au passé - nous auront toujours à nous confronter à ce dernier -, ouvrirait peut-être à un présent plus éclairé. En reprenant la réflexion de Didi-Huberman, la distanciation serait l'une des conditions de ce regard approfondi qui est la base d'un exercice de mémoire: "La distanciation est une opération de connaissance qui vise, par les moyens de l'art, une possibilité de regard critique sur l'histoire ${ }^{16}$. Mais comment trouver la bonne distance ? Si la résistance aux images impose une distance excessive, comment savoir où se trouvent les limites au- 
delà desquelles on risquerait une adhésion à l'image nous empêchant d'atteindre l'expérience esthétique tout court, puis l'élaboration d'une esthétique de la résistance ?

Une poétique de l'enregistrement irait dans le sens d'une élaboration des images de la résistance, ou de résistance. Les exemples abordés ici rendent compte de cette poétique, ils sont chargés aujourd'hui d'une certaine aura liée à leur production pendant la dictature. Les photographies des manifestations - comme celle de la foule avançant avec l'image d'une colombe de la paix - ou les photos des performances réalisées à l'époque - comme celles de Diamela Eltit et des Juments de l'Apocalypse -, ainsi que les photographies de la Cueca sola, coïncident dans une poétique de l'enregistrement qui les unit au-delà des énormes écarts qui les séparent. La photographie rassemble ces images, comme le fait la dictature - elles sont toutes nées dans ce contexte particulier. Or, au-delà des questions formelles, ce qui semble leur octroyer une telle valeur, c'est leur relative rareté et, si l'on ose dire, la pauvreté qui les caractérise : images en noir et blanc dont le négatif a parfois disparu, images de vidéo d'une moindre qualité, images réalisées avec peu de moyens techniques, etc.

31 La force de ces images résiderait donc dans la capacité des artistes, familles des victimes, citoyens engagés, etc., de faire appel à la créativité pour produire des images impressionnantes, qui disent ce qui est interdit de dire, qui critiquent et qui demeurent. Le manque de moyens serait remplacé par l'abondance d'« images" produites par une censure de l'expression, l'enjeu de faire image en dictature étant la liberté même. La justesse de l'image devenait une exigence. Ces photographies avaient donc une fonction politique, l'enregistrement était un acte politique. Mais, que reste-til aujourd'hui de la dimension politique de ces images? En quoi quelque chose de l'ordre de l'héritage se conserverait-il ou disparaitre-t-il ? Quel est l'enjeu de restituer à ces images leur aspect politique - c'est-à-dire de les désacraliser pour pouvoir les approcher, penser leurs vertus et leurs limites, se questionner sur les détails concernant leur réalisation, etc.? Bref, quelles sont les limites des archives et par rapport à quoi? En reprenant le questionnement de Jacques Derrida: "Mais où commence le dehors? Cette question est la question de l'archive. Il n'en est sans doute pas d'autre $»^{17}$.

Ces limites considéreraient, par exemple, une étude de l'apparition du corps de la femme - dont certaines de ces photos rendent compte - donnant visibilité à une image inédite dans le contexte artistique chilien : le corps féminin véhiculant l'expression de sa douleur et de sa solitude à travers une action spécifique, artistique. Les photographies de Diamela Eltit portant des traces évidentes de violences auto infligées ou celles des femmes des disparus réalisant la danse nationale - nous y retrouvons le recours à l'emblème national (drapeau, hymne, danse) utilisé de manière critique par des nombreux artistes en temps de dictature - ce qui nous rappelle que «la généalogie de l'attitude analytique, dont elle est un exemple très particulier, n'est pertinente que par rapport à la manière propre dont la photographie forme ses images ${ }^{18}$. L'attitude analytique présente dans ces photographies contraste pourtant avec le travail des musées et des centres culturels recueillant les archives de la mémoire. Ces derniers ont généré une circulation inouïe d'images de la dictature à travers des constructions qui vont des bâtiments monumentaux à l'architecture épurée jusqu'aux centres culturels installés dans des anciens locaux de torture ou de détention. Ces espaces présentent des dispositifs spécifiques invitant le public au recueillement et à la prise de conscience des graves violations des Droits de l'Homme produites dans le pays. Or, il nous semble que 
l'un des aspects les plus problématiques de ces démarches d' «administration » ou de gestion des images de la mémoire concerne la photographie. Elle est présente dans le travail d'archive et dans les exhibitions, dans des formats divers et avec des fonctions variées. Il serait donc incongru d'accuser son « oubli ». Cependant, il semble nécessaire de solliciter une mise en question et une mise en tension de ses usages. À quoi faisonsnous donc référence quand nous suggérons un certain oubli ?

Le problème des usages de la photographie dans une gestion de mise en images de la dictature est celui d'une résistance aux images. Les photos y apparaissent, elles sont visibles, mais elles ne sont pas accessibles. C'est-à-dire qu'elles ne sont pas là pour susciter un véritable travail de mémoire, mais pour proposer une administration du traumatisme par la voie de la remémoration et d'une certaine délivrance imaginale. Elles ne sont donc pas exploitées pour une esthétique qui les rendrait accessibles, "touchables", actives, problématiques. Elles acquièrent plutôt une fonction esthétisante dans ces lieux; elles ne sont certes pas des ornements, mais elles ne sont pas non plus des objets pour la pensée. Une absence de photographie contemporaine ayant la dictature par objet, nous semble être la conséquence directe de ce travail défaillant de la mémoire photographique de la période.

Ce dernier refuge pour la résistance identifié ici nous rappelle les transformations subies par le traitement de la résistance dans la théorie psychanalytique de Freud. Il passe ainsi d'un travail d'insistance se posant comme " une force de sens contraire à la

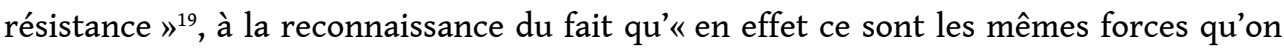
voit à l'œuvre dans la résistance et le refoulement $»^{20}$. Cette appréciation profonde de la portée de la résistance élargit la perspective d'une possible levée du refoulement. Dans ce cheminement, Freud observe la relation intrinsèque entre résistance et défense du moi : "Les mécanismes de défense contre des dangers anciens font retour dans la cure sous forme de résistances à la guérison, et cela parce que la guérison est elle-même considérée par le moi comme un nouveau danger $»^{21}$. Pour penser la question d'un regard qui se positionne et qui dépasse une résistance qui, d'une certaine manière, le préserverait d'une image insupportable, nous insisterons sur le développement qu'a eu ce concept dans la démarche théorique et pratique de son auteur.

Nous en arrivons ainsi à l'identification par Freud - dans le livre Inhibition, symptôme et angoisse - de cinq espèces de résistance. Nous nous concentrerons sur l'une d'elles : « La cinquième résistance, celle du sur-moi, celle qui a été reconnue la dernière, la plus obscure, mais pas toujours la plus faible, semble être issue de la conscience de culpabilité ou du besoin de punition; elle s'oppose à tout succès et en conséquence aussi à la guérison par l'analyse ${ }^{22}$. D'après cette révision succincte du terme, et en regard de l'évolution qu'il a eu au cours de la recherche de Freud, nous nous demandons si le problème de résistance à la photo de la dictature ne relèverait pas de cette cinquième résistance. Une étude de la censure exercée en dictature, mais aussi de l'autocensure ou du refoulement s'étendant au-delà de la période comme un vestige de cette atteinte à la liberté d'expression, apporterait des éléments pour penser une résistance du regard. Les photos de l'histoire, tout en restant visibles, ont eu peine à être considérées comme des objets pour la pensée. Les conséquences les plus marquantes de ce phénomène ont été une difficulté d'énonciation découlant d'une véritable appréciation esthétique, et un manque de création artistique visant une politique de l'image de la dictature. 

nombreuses manifestations et performances ont eu lieu. Celle qui attirera davantage notre attention et qui nous permettra de faire un lien avec les manifestations et les enregistrements étudiés ici, s'intitule Querer no ver. Elle n'a pas eu lieu le 11 mais le 10 septembre, et consistait en un appel lancé à travers les réseaux sociaux, invitant les passants à s'allonger sur le trottoir pendant 11 minutes, sans bouger, en faisant une ligne de corps, comme s'il s'agissait des corps morts. L'artiste et sémiologue Maria José Contreras, associée à un groupe d'artistes, a appelé à cette action massive qui a compté plus de mille participants. Plus précisément, 1210 personnes gisaient par terre, en donnant corps à cette action. La manifestation a attiré l'attention des passants - qui devaient parfois passer à travers les corps pour pouvoir continuer leur chemin -, mais aussi celle des médias.

regardant les images de ce travail réalisées et publiées par les médias, nous pouvons apprécier l'écart existant entre l'enregistrement tel que nous l'avons conçu dans ce chapitre vis-à-vis des actions des manifestants «en » dictature, et ce qu'on peut considérer comme une médiatisation de l'action. Les photographies apparues dans les médias ne rendent pas compte de l'étendue de l'action et, ce qui semble encore plus problématique, elles en réduisent parfois la portée politique. Selon la tendance du média, cette action a été décrite comme «La cicatrice humaine qui a poussé dans le centre de Santiago ${ }^{23}$ ou comme «La longue file de corps allongés pour se souvenir des disparus $»^{24}$. Or, l'énorme quantité d'images circulant sur Internet n'engageait pas forcément un œil performant, comme le faisaient les actions étudiées plus haut. On voit ainsi comment, d'une part, cette démarche reprend l'idée des manifestations d'une autre époque, et, d'autre part, elle en diffère par un travail d'enregistrement extérieur, plus concentré sur l'anecdote que sur lui-même. En résumé, bien que cette action ait eu une visibilité importante dans les médias, elle n'intègre pas dans sa conception l'élément fondamental de l'enregistrement photographique. Une poétique de l'enregistrement n'aura par conséquent pas caractérisé ces images.

\section{BIBLIOGRAPHY}

Derrida Jacques, Mal d'archive, Paris, Galilée, 1995.

Didi-Huberman Georges, Quand les images prennent position. L'œil de l'histoire 1, Paris, Minuit, 2009.

Didi-Huberman Georges, Remontages du temps subi. L'œil de l'histoire 2, Paris, Minuit, 2010.

Eltit Diamela, Lumpérica, Santiago de Chile, Ornitorrinco, 1983.

Freud Sigmund, Inhibition, symptôme et angoisse, traduit de l'allemand par Joël et Roland Doron, Paris, Presses universitaires de France, 1993.

Krauss Rosalind, Le photographique. Pour une théorie des écarts, Paris, Macula, 1990.

Laplanche Jean, Pontalis Jean-Bertrand, Vocabulaire de la psychanalyse, Paris, Presses universitaires de France, 1967.

Appareil, 14 | 2014 
Rancière Jacques, Le destin des images, Paris, La Fabrique, 2003.

Soulages François, Esthétique de la photographie. La perte et le reste, Paris, Nathan, 1998.

\section{NOTES}

1. François Soulages, Esthétique de la photographie. La perte et le reste, Paris, Nathan, 1998, p. 281.

2. Diamela Eltit, Lumpérica, Santiago de Chile, Ornitorrinco, 1983.

3. Nous pensons notamment aux performances de Raul Zurita, ainsi qu'aux performances et happenings du collectif Las yeguas del apocalipsis, où la dimension sacrificielle de la blessure physique joue aussi un rôle central.

4. Georges Didi-Huberman, Remontages du temps subi. L'œil de l'histoire 2, Paris, Minuit, 2010, p. 24.

5. Ibid., p. 5.

6. Diamela Eltit, Zona de dolor (Zone de douleur), performance, Santiago du Chili, 1980.

7. Jacques Derrida, Mal d'archive, Paris, Galilée, 1995, p. 14.

8. Ibid., p. 15.

9. Jacques Rancière, Le destin des images, Paris, La Fabrique, 2003, p. 25.

10. Téléanalisis était la «version télévisuelle » de la revue Analisis média de gauche apparu sous la dictature et qui était confronté systématiquement à la censure, à l'emprisonnement de ses journalistes et photographes. Il consistait en des journaux informatifs en VHS qui circulaient de main en main ; ils ont été conservés et ils font partie des archives utilisées pour l'émission Chili, les images interdites.

11. Sebastian Moreno, La ciudad de los fotógrafos [La Cité des photographes], documentaire, 80 min, 2006.

12. François Soulages, Esthétique de la photographie. La perte et le reste, Paris, Nathan, 1998, p. 139.

13. Association grémial créée le 19 juin 1981 pour donner une protection aux photoreporters. Elle a comme but aussi de professionnaliser le métier de photographe.

14. Le référendum chilien du 5 octobre 1988 visait à décider le maintien (ou non) du général Augusto Pinochet au pouvoir jusqu'en 1997. La population était ainsi appelée à se prononcer pour le « oui » ou pour le « non » à la dictature.

15. Georges Didi-Huberman, Quand les images prennent position. L'œil de l'histoire 1, Paris, Minuit, 2009 , p. 68.

16. Ibid., p. 69.

17. Jacques Derrida, Mal d'archive, Paris, Galilée, 1995, p. 20.

18. Rosalind Krauss, Le photographique. Pour une théorie des écarts, Paris, Macula, 1990, p. 35.

19. Jean Laplanche et Jean-Bertrand Pontalis, Vocabulaire de la psychanalyse, Paris, Presses universitaires de France, 1967, p. 420.

20. Ibid.

21. Ibid., p. 421.

22. Sigmund Freud, Inhibition, symptôme et angoisse, traduit de l'allemand par Joël et Roland Doron, Paris, Presses universitaires de France, 1993, p. 72.

23. Patricio Gonzalez Rios, "La cicatriz humana que broto en el centro de Santiago ", El mostrador, 13/09/2013.

24. Esteban Garay, «La Larga fila de cuerpos tendidos para recordar a desaparecidos », La Nacion, 10/09/2013. 


\section{ABSTRACTS}

Pour penser d'une part les frontières de l'enregistrement, et d'autre part les possibilités de l'archive photographique de la dictature, nous approfondirons les usages d'une certaine photographie qui a pour objet les manifestations aussi bien politiques qu'artistiques accomplies par les familles des victimes, par les artistes et par les citoyens engagés contre la dictature au Chili. La performance artistique et les manifestations de contestation sont des événements d'ordre différent. Cependant, la photographie joue un rôle essentiel dans les deux cas : elle rend « visibles » ces actions dont la présence médiatique est nulle à cause de la censure.

INDEX

Mots-clés: dictature, esthétique, photographie, résistance, performance, archive, Chili

\section{AUTHOR}

\section{JAVIERA MEDINA}

Docteur de l'université Paris 8, laboratoire EPHA, Esthétique, javieraml@gmail.com 